1 Instituto de Medicina Integral Professor Fernando Figueira (Imip), Grupo de Estudos de Gestão e Avaliação em Saúde (Geas) - Recife (PE), Brasil. cassandra-costa@hotmail. com

2 Instituto de Medicina Integral Professor Fernando Figueira (Imip), Grupo de Estudos de Gestão e Avaliação em Saúde (Geas) - Recife (PE), Brasil. Secretaria Estadual de Saúde de Pernambuco Recife (PE), Brasil. aymee.assessoriasanar@ gmail.com

3 Instituto de Medicina Integral Professor Fernando Figueira (Imip), Grupo de Estudos de Gestão e Avaliação em Saúde (Geas) - Recife (PE), Brasil. grecisoares@gmail.com

4 Instituto de Medicina Integral Professor Fernando Figueira (Imip), Grupo de Estudos de Gestão e Avaliação em Saúde (Geas) - Recife (PE), Brasil. rpfjesus@gmail.com

5 Instituto de Medicina Integral Professor Fernando Figueira (Imip), Grupo de Estudos de Gestão e Avaliação em Saúde (Geas) - Recife (PE), Brasil. ana_albuquerque1@hotmail. com

\section{Programa de Controle da Esquistossomose: avaliação da implantação em três municípios da Zona da Mata de Pernambuco, Brasil}

\author{
Schistosomiasis Control Program: evaluation of the implantation in \\ three municipalities of the Zona da Mata of Pernambuco, Brazil
}

Cassandra de Sousa Costa $\mathbf{1}$, Aymée Medeiros da Rocha ${ }^{\mathbf{2}}$, Greciane Soares da Silva ${ }^{\mathbf{3}}$, Renata Patrícia Freitas Soares de Jesus ${ }^{4}$, Ana Coelho de Albuquerque ${ }^{\mathbf{5}}$

RESUMO O presente estudo teve como objetivo avaliar a implantação das ações do Programa de Controle da Esquistossomose (PCE) em três municípios da Mata Sul de Pernambuco. Trata-se de uma avaliação normativa, com a realização de entrevistas semiestruturadas com profissionais da vigilância em saúde e da atenção básica. Os resultados encontrados demonstram que os municípios estudados obtiveram o grau não implantado. Como pontos críticos, observaram-se deficiências na gestão e na execução de ações de educação em saúde. Porém, é importante ressaltar que, nos três municípios avaliados, foi possível constatar um envolvimento relevante da atenção básica nas ações do PCE.

PALAVRAS-CHAVE Avaliação em saúde. Esquistossomose. Doenças negligenciadas. Vigilância em saúde pública. Atenção Primária à Saúde.

ABSTRACT The present study aimed to evaluate the implantation of Schistosomiasis Control Program (PCE) actions in three municipalities of Zona da Mata, state of Pernambuco. A normative evaluation was conducted, and semi-structured interviews were carried out with health surveillance and primary health care professionals. The results found show that the studied municipalities obtained the degree of not implemented. The most critical points identified were related to health education activities and management deficiencies. However, it is important to highlight that, in the three municipalities evaluated, it was possible to perceive a relevant involvement of primary care in the PCE's actions.

KEYWORDS Health evaluation. Schistosomiasis. Neglected diseases. Surveillance in public health. Primary Health Care. 


\section{Introdução}

De acordo com a Organização Mundial da Saúde (OMS), estima-se que a esquistossomose acometa cerca de 200 milhões de pessoas em todo o mundo (WORLD HEALTH ORGANIZATION, 1985), das quais, 7 milhões de infectados encontram-se no Brasil, país que apresenta altas prevalências e expansão das áreas de ocorrência dessa parasitose (KATZ; PEIXOTO, 2000; ROLLEMBERG ET AL., 2011).

O surgimento de um programa de controle específico para a doença, no Brasil, ocorreu em 1975, com a criação do Programa Especial de Controle da Esquistossomose (Pece), pela Superintendência de Campanhas de Saúde Pública (Sucam), sendo substituído, posteriormente, pelo Programa de Controle da Esquistossomose (PCE), na década de 1980. Com a descentralização das ações de vigilância e controle de doenças, em 1999, a execução das ações do PCE passou a ser de responsabilidade compartilhada, e os municípios passaram a exercer um papel fundamental (BRASIL, 1999; FAVRE ET AL., 2001).

Uma série de ações, visando à correta realização das atividades do PCE, deve ser realizada em âmbito municipal, como delimitação demográfica, inquéritos coproscópicos censitários, tratamento de infectados, controle de planorbídeos, medidas de saneamento ambiental, educação em saúde, vigilância epidemiológica e alimentação anual do Sistema de Informação do PCE (SISPCE). Essas atividades devem ser incorporadas à rotina desenvolvida pela Estratégia Saúde da Família (ESF) (BRASIL, 2004; BRASIL, 2008).

O estado de Pernambuco ainda é considerado a Unidade Federada do Brasil com maior grau de endemicidade para a esquistossomose, apresentando, no ano de 2015, uma positividade de 3,0\% entre as 242.419 pessoas examinadas. Entre os anos de 2005 e 2014, o estado apresentou uma média de 181 óbitos por ano, demonstrando, nesse mesmo período, a maior taxa de mortalidade quando comparada à da região Nordeste e à do País (Pernambuco, 2015). Tendo em vista esse cenário de gravidade, a Secretaria Estadual de Saúde de Pernambuco implantou, em 2011, o Programa Sanar de enfrentamento das doenças negligenciadas, visando a reduzir ou eliminar, enquanto problema de saúde pública, sete doenças transmissíveis negligenciadas, as quais apresentavam indicadores inaceitáveis, de acordo com a Organização Pan-Americana da Saúde e a OMS (2009), entre elas, a esquistossomose.

As ações de controle da esquistossomose realizadas pelo Programa Sanar foram desenvolvidas em 40 municípios prioritários, que apresentavam uma prevalência média maior que $10,0 \%$ entre os anos de 2005 e 2010. Entre as abordagens utilizadas por esse programa está o tratamento seletivo (TS), que objetiva o envolvimento efetivo nas atividades de vigilância, diagnóstico e tratamento dos casos positivos por parte dos profissionais das equipes da ESF, por meio da oferta de exames parasitológicos, assim como de exames clínicos e acompanhamento dos casos positivos por parte dos enfermeiros e médicos (PERNAMBUCO, 2012A).

Apesar de haver uma escolha de municípios prioritários para a realização das ações do Programa de maneira mais direcionada, sabe-se que outros municípios do estado possuem localidades endêmicas e que o enfrentamento da esquistossomose nesses municípios também é importante. Dessa forma, o presente estudo visa a avaliar o grau de implantação das ações do PCE em três municípios não prioritários para o Programa Sanar do estado de Pernambuco, e que utilizam a estratégia do tratamento seletivo para controle da esquistossomose.

\section{Métodos}

Realizou-se uma avaliação normativa, com abordagem quantitativa, para os 
componentes de estrutura e processo das ações de controle da esquistossomose em três municípios não prioritários para o programa Sanar do estado de Pernambuco. Para tanto, foi utilizada a abordagem de estrutura e processo proposta por Donabedian (1966), que se preocupa em saber em que medida as ações realizadas pelo programa são adequadas para alcançar os objetivos propostos para um efetivo controle. Algumas razões justificam a necessidade de se combinar a análise da estrutura e do processo nos estudos avaliativos. Entre elas, a possibilidade de se conduzir uma avaliação mais ampla, tendo em vista que certas categorias de informação podem ser mais indicativas de aspectos específicos do cuidado, além de aumentar a confiança na validade dessas conclusões, visto que as inferências são retiradas de vários tipos de indicadores (DONABEDIAN, 1981).

Apesar de o presente estudo ter sido realizado em municípios não prioritários, optou-se por analisar o grau de implantação (GI) das ações de controle da esquistossomose utilizando como base as ações preconizadas pelo Programa Sanar, que tem utilizado, pioneiramente, no estado de Pernambuco, estratégias de articulação entre vigilância em saúde e atenção básica com a finalidade de subsidiar o aprimoramento das ações de controle da doença, melhoria da assistência e dos serviços prestados à população.

O estudo foi realizado em duas etapas: na primeira, foi construído o modelo lógico do PCE, a partir de análise documental das seguintes publicações oficiais relativas ao programa: (i) 'Caderno de Atenção Básica $\mathrm{n}^{0}$ 21' (BRASIL, 2008); (ii) 'Política Nacional de Atenção Básica' (BRASIL, 2006); (iii) 'Vigilância da Esquistossomose Mansoni: diretrizes técnicas' (BRASIL, 2014); (iv) Portaria ${ }^{\circ}$ 1.172, de 15 de junho de 2004 (BRASIL, 2004); (v) 'Guia operacional para redução da Esquistossomose em Pernambuco' (2012A); (vi) 'Guia Operacional do SISPCE' (2012B) (figura 1). O delineamento do modelo lógico do programa se fez em cinco componentes: gestão, vigilância epidemiológica, apoio laboratorial, atenção à saúde e educação em saúde. 
Figura 1. Modelo lógico das ações de controle da esquistossomose. Pernambuco, 2015

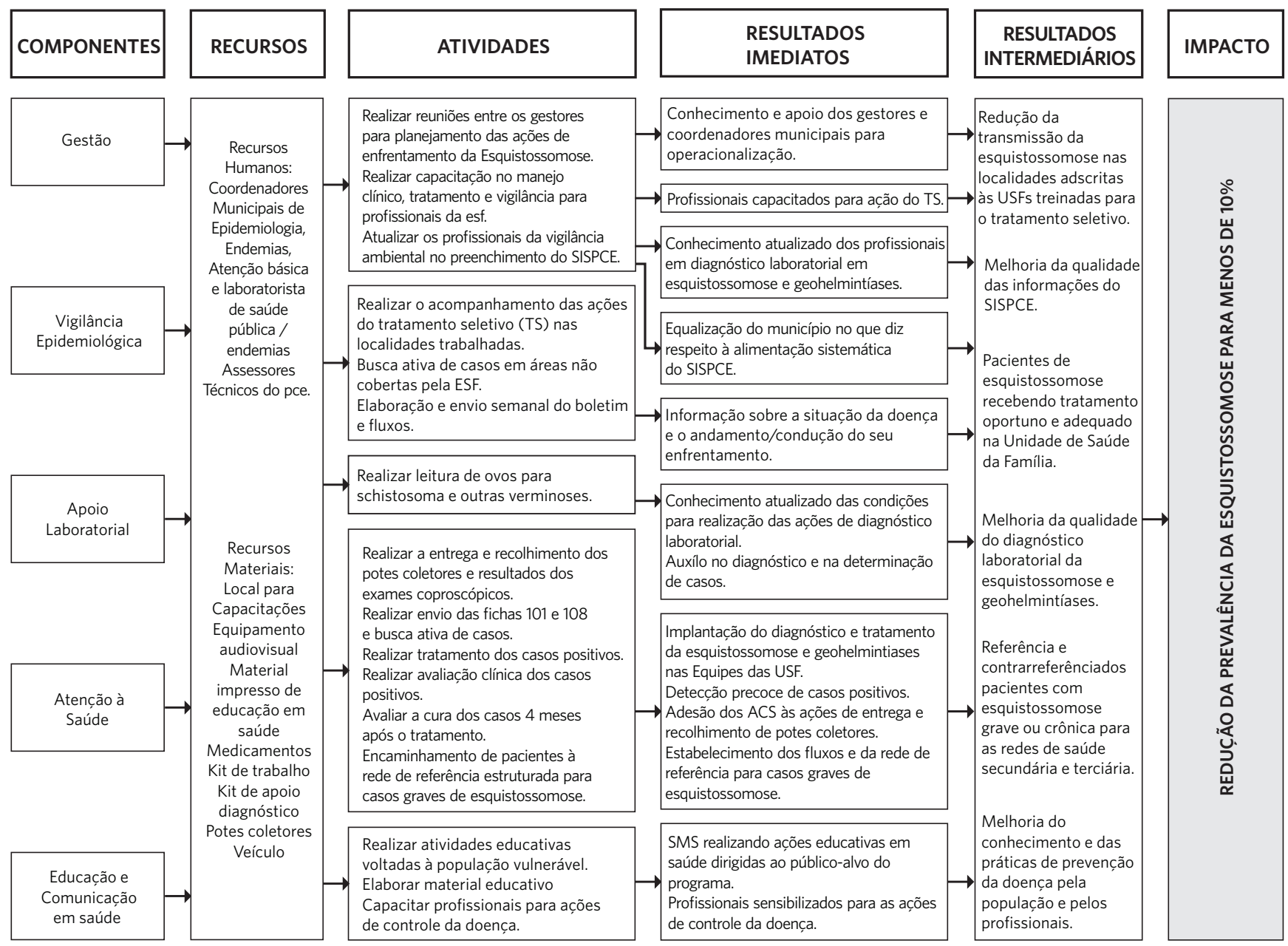

A segunda etapa do estudo consistiu na aferição do GI do PCE. A partir do modelo lógico, foi elaborada a matriz de análise e julgamento, a qual, posteriormente, foi submetida a um comitê de especialistas, formados por um profissional da área de avaliação e dois do serviço, ambos com experiência em esquistossomose no âmbito do Sistema Único de Saúde (SUS). A matriz foi construída com duas dimensões relacionadas à estrutura e ao processo, cada uma com 100 pontos, distribuídos em 21 critérios de estrutura e 27 critérios de processo. Para sua consolidação, foi realizada uma reunião com os pesquisadores e, por meio de comparação critério a critério, foram incorporadas as sugestões enviadas pelos especialistas.

Para a avaliação do GI, foi aplicado um questionário estruturado, elaborado a partir do modelo lógico e da matriz, direcionado aos gestores de vigilância em saúde e atenção básica e Agentes de Combate às Endemias (ACE), além de médicos, enfermeiros e Agentes Comunitários de Saúde (ACS) de 7 Unidades de Saúde da Família (USF), selecionadas por estarem inseridas em áreas de risco de transmissão da doença e que foram capacitadas para desempenhar as atividades 
do tratamento seletivo da esquistossomose. No total, o questionário foi aplicado com 25 entrevistados, distribuídos entre os três municípios da pesquisa.

Para quantificar a realização das atividades, foi empregado um sistema de escores utilizado por Quinino e colaboradores (2010). Dessa forma, a pontuação alcançada pela estrutura (E) recebeu peso quatro; e, pelo processo $(\mathrm{P})$, peso seis. A fórmula para cálculo do GI foi: GI = E (x 4) + P (x 6): 10. O GI do PCE foi classificado em três situações: 'implantado' = 90 a 100 pontos; 'parcialmente implantado' = 60 a 89 pontos; e 'não implantado' $<59$ pontos.

O estudo foi aprovado pelo Comitê de Ética em Pesquisa em Seres Humanos do Instituto de Medicina Integral Professor Fernando Figueira (Imip), conforme CAAE $n^{\circ}$ 34547914.8.0000.5201, em reunião ordinária ocorrida no dia 10 de setembro de 2014 , estando de acordo com a resolução 466/12 do Conselho Nacional de Saúde.

\section{Resultados}

Pode-se observar que houve a inserção de atividades instituídas pelo Programa Sanar nas atividades de rotina do PCE nos municípios estudados, para os componentes Gestão, Vigilância Epidemiológica e Atenção à Saúde, tais como: i) realizar capacitação no manejo clínico, tratamento e vigilância para profissionais da ESF; ii) realizar o acompanhamento das ações do TS nas localidades trabalhadas; iii) realizar a entrega e o recolhimento dos potes coletores e resultados dos exames coproscópicos.

Os resultados quanto à estrutura e ao processo não obtiveram grandes variações entre os três municípios da pesquisa. Os GI do PCE nos municípios I, II e III foram de $26,31 \%, 36,3 \%$ e $24,77 \%$, respectivamente, sendo todos classificados como GI 'não implantado'. Os resultados da análise do GI da dimensão estrutura encontram-se na tabela 1.

Tabela 1. Distribuição da pontuação atingida em cada subcomponente da dimensão estrutura nos municípios I, II e III, 2015

\begin{tabular}{|c|c|c|c|c|c|}
\hline Componente & Critério & $\begin{array}{l}\text { Pontuação } \\
\text { Máxima do } \\
\text { Critério }\end{array}$ & $\begin{array}{l}\text { Pontuação } \\
\text { Atingida MI }\end{array}$ & $\begin{array}{l}\text { Pontuação } \\
\text { Atingida MII }\end{array}$ & $\begin{array}{l}\text { Pontuação } \\
\text { Atingida MII }\end{array}$ \\
\hline \multirow{4}{*}{ Gestão } & Existência de profissional - gestão em saúde & 2,4 & 2,4 & 2,4 & 2,4 \\
\hline & Recursos humanos qualificados para as ações do PCE & 2,4 & 0,9 & 0,9 & 0 \\
\hline & Disponibilidade de veículo para as ações do PCE & 2,4 & 0 & 0 & 0 \\
\hline & TOTAL DE PONTOS & $7,2(100,0 \%)$ & $3,3(45,8 \%)$ & $3,3(45,8 \%)$ & $2,4(33,3 \%)$ \\
\hline \multirow{6}{*}{$\begin{array}{l}\text { Vigilância } \\
\text { epidemioló- } \\
\text { gica e am- } \\
\text { biental }\end{array}$} & Existência de profissional - gestão em saúde & 2,4 & 0 & 2,4 & 0 \\
\hline & $\begin{array}{l}\text { Recursos humanos suficientes para as ações de controle de } \\
\text { esquistossomose }\end{array}$ & 2,4 & 0 & 0 & 0 \\
\hline & Recursos humanos qualificados para as ações do PCE & 2,4 & 0,6 & 2,4 & 1,8 \\
\hline & Disponibilidade de computador com SISPCE instalado & 2,4 & 2,4 & 2,4 & 2,4 \\
\hline & Digitador capacitado & 2,4 & 2,4 & 2,4 & 0 \\
\hline & TOTAL DE PONTOS & $12(100,0 \%)$ & $5,4(45,0 \%)$ & $9,6(80,0 \%)$ & $4,2(35,0 \%)$ \\
\hline
\end{tabular}


Tabela 1. (cont.)

\begin{tabular}{|c|c|c|c|c|c|}
\hline \multirow{4}{*}{$\begin{array}{l}\text { Apoio labora- } \\
\text { torial }\end{array}$} & $\begin{array}{l}\text { Recursos humanos suficientes para as ações de controle da } \\
\text { esquistossomose }\end{array}$ & 2,4 & 2,4 & 2,4 & 0 \\
\hline & Recursos humanos qualificados para as ações do PCE & 2,4 & 2,4 & 2,4 & 2,0 \\
\hline & $\begin{array}{l}\text { Recursos materiais para a realização dos exames coproscó- } \\
\text { picos }\end{array}$ & 2,4 & 2,4 & 2,4 & 2,4 \\
\hline & TOTAL DE PONTOS & $7,2(100,0 \%)$ & $7,2(100,0 \%)$ & $7,2(100,0 \%)$ & $4,4(61,0 \%)$ \\
\hline \multirow{8}{*}{$\begin{array}{l}\text { Atenção à } \\
\text { saúde }\end{array}$} & Existência de profissional - gestão em saúde & 2,4 & 1,92 & 1,92 & 1,92 \\
\hline & $\begin{array}{l}\text { Existência de material para os profissionais desenvolverem as } \\
\text { ações de controle da esquistossomose disponibilizado pela } \\
\text { Secretaria Municipal de Saúde (SMS) }\end{array}$ & 2,4 & 0 & 0 & 2,4 \\
\hline & Presença das fichas utilizadas para as ações do PCE & 2,4 & 2,4 & 2,4 & 0 \\
\hline & $\begin{array}{l}\text { Disponibilidade de medicação para o tratamento dos casos } \\
\text { positivos }\end{array}$ & 2,4 & 2,4 & 2,4 & 0 \\
\hline & Presença de balança de chão & 2,4 & 2,4 & 2,4 & 2,4 \\
\hline & $\begin{array}{l}\text { Disponibilidade de recursos materiais para atendimento de } \\
\text { urgências }\end{array}$ & 2,4 & 2,4 & 2,4 & 2,4 \\
\hline & Recursos humanos qualificados para as ações do PCE & 2,4 & 1,35 & 1,95 & 1,95 \\
\hline & TOTAL DE PONTOS & $16,8(100,0 \%)$ & $12,87(76,6 \%)$ & $13,47(80,0 \%)$ & $11,07(65,8 \%)$ \\
\hline \multirow{4}{*}{$\begin{array}{l}\text { Educação em } \\
\text { saúde }\end{array}$} & Recursos materiais para as ações de educação & 2,4 & 2,4 & 2,4 & 0 \\
\hline & Recursos materiais para as ações de mobilização social & 2,4 & 0 & 0 & 0 \\
\hline & Equipe de educação em saúde & 2,4 & 2,4 & 0 & 0 \\
\hline & TOTAL DE PONTOS & $7,2(100,0 \%)$ & $4,8(66,6 \%)$ & $2,4(33,3 \%)$ & 0,0 \\
\hline
\end{tabular}

Os municípios apresentaram alguns aspectos favoráveis relacionados à 'estrutura', tais como laboratório estruturado para análise das lâminas (100,0\%, 100,0\% e 61,5\%), existência de coordenação do PCE $(100,0 \%, 100,0 \%$ e $100,0 \%)$ e da atenção básica $(80,0 \%, 80,0 \%$ e $80,0 \%)$ capacitados para gerir as ações do programa, bem como computador com SISPCE e internet instalados para o envio dos dados (100,0\%, 100,0\% e 100,0\%) (tabela 1).

É possível identificar que os pontos críticos relacionados a esse componente são a disponibilidade de veículo para as ações do PCE, recursos humanos suficientes para as ações de controle da esquistossomose, bem como recursos materiais para as ações de mobilização social, os quais contribuíram para as condições insatisfatórias da dimensão estrutura. Além disso, apenas o MI fez referência à existência de equipe de educação em saúde (100,0\%), e o MII à existência de gestor da vigilância epidemiológica e ambiental (100,0\%). Já no MIII, foi possível observar a falta de recursos humanos qualificados para as ações do PCE, de digitador capacitado, de medicação para o tratamento dos casos positivos, das fichas utilizadas para as ações do PCE e de recursos materiais para as ações de educação em saúde $(0,0 \%$ para todos) (tabela 1).

No que diz respeito aos resultados da 
análise do GI da dimensão 'processo' nos municípios MI, MII e MIII, os mesmos encontram-se na tabela 2. Todos foram considerados como GI 'não implantado', com $50,0 \%, 58,2 \%$ e $50,0 \%$, respectivamente.

Tabela 2. Distribuição da pontuação atingida em cada subcomponente da dimensão processo nos municípios I, II e III, 2015

\begin{tabular}{|c|c|c|c|c|c|}
\hline Componente & Critério & $\begin{array}{l}\text { Pontuação } \\
\text { Máxima do } \\
\text { Critério }\end{array}$ & $\begin{array}{l}\text { Pontuação } \\
\text { Atingida MI }\end{array}$ & $\begin{array}{l}\text { Pontuação } \\
\text { Atingida MII }\end{array}$ & $\begin{array}{l}\text { Pontuação } \\
\text { Atingida MII }\end{array}$ \\
\hline \multirow{3}{*}{ Gestão } & $\begin{array}{l}\text { Realização de reuniões entre os gestores para apresentação } \\
\text { de estratégias e pactuação das ações de enfrentamento da } \\
\text { esquistossomose. }\end{array}$ & 1,85 & 0 & 1,85 & 1,85 \\
\hline & $\begin{array}{l}\text { Realização de capacitação para os profissionais em suas } \\
\text { áreas específicas: Coordenador do PCE e atenção básica, } \\
\text { profissionais de nível superior, ACS, ACE, técnico e auxiliar } \\
\text { do laboratório e digitador do PCE. }\end{array}$ & 1,85 & 0,46 & 0 & 0,46 \\
\hline & TOTAL DE PONTOS & $3,7(100,0 \%)$ & $0,46(12,4 \%)$ & $1,85(50,0 \%)$ & $2,3(62,4 \%)$ \\
\hline \multirow{10}{*}{$\begin{array}{l}\text { Vigilância } \\
\text { epidemioló- } \\
\text { gica e am- } \\
\text { biental }\end{array}$} & Elaboração de Boletim Informativo & 1,85 & 0 & 0 & 0 \\
\hline & Digitação no SISPCE & 1,85 & 1,85 & 1,85 & 1,85 \\
\hline & Busca ativa de casos em áreas não cobertas pela ESF & 1,85 & 0 & 1,85 & 1,85 \\
\hline & $\begin{array}{l}\text { Notificação e investigação de casos graves no Sistema de } \\
\text { Informação de Agravos de Notificação (Sinan) }\end{array}$ & 1,85 & 1,6 & 1,85 & 0,23 \\
\hline & Utilização da informação & 1,85 & 1,85 & 1,85 & 1,85 \\
\hline & Distribuição dos potes pelos ACE & 1,85 & 0 & 1,85 & 1,85 \\
\hline & Recolhimento dos potes pelos ACE & 1,85 & 0 & 1,85 & 1,85 \\
\hline & Acompanhamento dos pacientes em tratamento pelo ACE & 1,85 & 0 & 1,85 & 1,85 \\
\hline & Realização de atividades de malacologia & 1,85 & 0 & 0 & 0 \\
\hline & TOTAL DE PONTOS & $16,65(100,0 \%)$ & $5,3(31,4 \%)$ & $12,95(77,7 \%)$ & $11,33(68,2 \%)$ \\
\hline \multirow{3}{*}{$\begin{array}{l}\text { Apoio labora- } \\
\text { torial }\end{array}$} & Leitura de lâminas para diagnóstico da esquistossomose & 1,85 & 0 & 0 & 0 \\
\hline & Envio das lâminas para controle de qualidade. & 1,85 & 0 & 1,85 & 0 \\
\hline & TOTAL DE PONTOS & $3,7(100,0 \%)$ & 0 & $1,85(50,0 \%)$ & 0 \\
\hline \multirow{10}{*}{$\begin{array}{l}\text { Atenção à } \\
\text { saúde }\end{array}$} & Distribuição dos potes coletores pelos ACS & 1,85 & 0 & 1,85 & 1,85 \\
\hline & Recolhimento de potes coletores pelos ACS & 1,85 & 1,85 & 1,85 & 1,85 \\
\hline & Participação dos ACS no inquérito coproscópico & 1,85 & 1,85 & 1,85 & 1,85 \\
\hline & Realização do inquérito coproscópico & 1,85 & 0 & 1,85 & 1,85 \\
\hline & Cobertura do inquérito (exames) & 1,85 & 0,46 & 0,92 & 0,92 \\
\hline & Realização de exames complementares & 1,85 & 1,85 & 0 & 0 \\
\hline & $\begin{array}{l}\text { Entrega de resultados dos exames coproscópicos (positivos } \\
\text { e negativos) pela ESF }\end{array}$ & 1,85 & 1,85 & 0,92 & 1,85 \\
\hline & Envio das fichas 101 e 108 para a vigilância epidemiológica & 1,85 & 0 & 1,85 & 0 \\
\hline & Tratamento dos casos positivos & 1,85 & 1,85 & 1,85 & 0 \\
\hline & Avaliação de cura dos casos positivos a cada 4 a 6 meses & 1,85 & 1,85 & 1,85 & 0 \\
\hline
\end{tabular}


Tabela 2. (cont.)

\begin{tabular}{|c|c|c|c|c|c|}
\hline \multirow{2}{*}{$\begin{array}{l}\text { Atenção à } \\
\text { saúde }\end{array}$} & $\begin{array}{l}\text { Encaminhamento de pacientes com formas graves da } \\
\text { doença para a referência }\end{array}$ & 1,85 & 1,38 & 1,85 & 0 \\
\hline & TOTAL DE PONTOS & $20,35(100,0 \%)$ & $12,94(63,6 \%)$ & $16,64(81,8 \%)$ & $10,17(50,0 \%)$ \\
\hline \multirow{4}{*}{$\begin{array}{l}\text { Educação em } \\
\text { saúde }\end{array}$} & Realização de atividades educativas por parte da SMS & 1,85 & 1,85 & 1,85 & 1,85 \\
\hline & $\begin{array}{l}\text { Realização, por parte dos profissionais da ESF, de ações de } \\
\text { educação em saúde e mobilização social junto à sociedade }\end{array}$ & 1,85 & 0,92 & 1,38 & 0,92 \\
\hline & $\begin{array}{l}\text { Distribuição de materiais para as ações de educação em } \\
\text { saúde }\end{array}$ & 1,85 & 0 & 0 & 0 \\
\hline & TOTAL DE PONTOS & $5,55(100,0 \%)$ & $2,77(50,0 \%)$ & $3,23(58,2 \%)$ & $2,77(50,0 \%)$ \\
\hline
\end{tabular}

O MII apresentou um desempenho superior na dimensão 'processo', se comparado aos demais, com os maiores resultados no que se refere aos componentes 'atenção à saúde' $(81,8 \%$ - componente parcialmente implantado) e 'vigilância epidemiológica e ambiental' (77,7\% - componente parcialmente implantado).

Com relação às atividades mais críticas, as seguintes apresentaram o resultado de 0,0\%: elaboração de boletim informativo, realização de atividades de malacologia, leitura de lâminas para diagnóstico da esquistossomose e distribuição de materiais para as ações de educação em saúde. Os três municípios estudados apresentaram fragilidades no componente 'apoio laboratorial', principalmente por não realizarem a leitura do número mínimo de lâminas semanal, conforme preconizado.

\section{Discussão}

Este estudo avaliativo das ações de controle da esquistossomose em três municípios não prioritários no estado de Pernambuco comparou a estrutura e o processo desenvolvido com critérios e normas estabelecidos pelo Ministério da Saúde e pelo Programa Sanar. Partiu-se da premissa de que o desenho da política de controle da esquistossomose, desenvolvido pelo Ministério da Saúde e adaptado pelo Programa Sanar, tem encontrado bons resultados nos municípios prioritários do estado.

Os achados desta pesquisa apontam problemas no componente 'educação em saúde', considerado 'não implantado' na dimensão 'estrutura' nos MII e MII, e na dimensão 'processo' nos três municípios (tabela 1 e tabela 2), o que pode revelar a ausência de prioridade das gestões municipais quanto às suas atividades. A indisponibilidade de recursos materiais voltados às atividades de educação em saúde prejudica a divulgação junto à população de informações sobre a prevenção e os fatores de risco para a doença, o que contribui diretamente para a permanência da transmissão. Outros estudos têm demonstrado a relação entre a escassez de recursos materiais e a carência no desenvolvimento de atividades educativas (PEREIRA; SERVO, 2006; DIAS; LOPES, 2013).

O processo de educação em saúde é uma das formas de se promover a saúde por meio do compartilhamento de saberes na busca de soluções dos mais diversos problemas. As atividades de educação em saúde devem ser realizadas independentemente dos níveis de prevalência da doença e visa não só a envolver as comunidades na execução das ações de controle, como, também, ampliar seu nível de conscientização, de modo a trazer o 
empoderamento necessário para demandar das autoridades competentes as medidas pertinentes para a solução do problema da esquistossomose (FARIAS, 2010).

No que tange ao componente 'apoio laboratorial', observou-se que, apesar de os municípios possuírem uma boa estrutura, a leitura de lâminas para diagnóstico da esquistossomose e o envio das lâminas para controle de qualidade não estão sendo realizados. A existência de recursos materiais básicos para a realização dos exames é de grande importância no PCE, pois sua ausência prejudica o diagnóstico e, consequentemente, o conhecimento da prevalência no município. A falha nesse componente, relativa ao processo, contribui para a não realização das atividades mínimas propostas para o controle da esquistossomose, o que interfere diretamente na persistência de indivíduos positivos e com um alto grau de infestação (QUININO ET AL., 2010).

Outro ponto crítico observado neste estudo se refere a problemas na coordenação da informação entre os setores. A não consolidação das informações, mediante a construção de boletins epidemiológicos, por exemplo, pode repercutir no monitoramento contínuo do programa e na utilização dessas informações para o planejamento das ações, a racionalização de recursos humanos e materiais, bem como para uma melhor articulação intrassetorial. Tais práticas permitem a tomada de decisão de maneira pertinente e que, acredita-se, contribuem para o alcance dos objetivos desejados. A não realização dessas atividades compromete todo o percurso das ações de controle da esquistossomose na instância municipal, uma vez que os profissionais ficam impossibilitados de equacionar as ações nas áreas com potencial de transmissão diferenciadas (QUININO ET AL., 2010).

Um resultado importante do estudo que necessita ser ressaltado é a efetiva participação dos ACS nas ações do PCE, tais como distribuição e recolhimento dos potes e participação nos inquéritos coproscópicos, o que demonstra a incorporação desse profissional nas atividades do programa e a integração das ações da vigilância e da atenção básica. Esses achados estão de acordo com a normatização vigente, a qual preconiza que o planejamento e a execução das ações de controle da esquistossomose devem ser realizados em conjunto entre as equipes do Programa de Agentes Comunitários de Saúde (Pacs)/Programa Saúde da Família (PSF) e da vigilância em saúde. Estudo realizado por Cesarino e colaboradores (2014) corrobora os resultados encontrados quando afirma que a experiência de integração do ACS com a vigilância é uma possibilidade a ser considerada, a qual pode evitar a fragmentação decorrente da forma como os serviços de controle de endemias desenvolvem suas ações atualmente.

No entanto, essa não é a realidade encontrada em outros estudos que analisaram a implementação de programas de controle da esquistossomose e da dengue, por exemplo, os quais apontaram para dificuldades na comunicação, descompasso entre as atribuições e desempenho de tarefas e para a falta de entrosamento entre as equipes de vigilância e atenção básica (OMOTTO; SANTINI; ESTEVES, 2009; MENEZES, 2005; CHIARAVALLOTI NETO, 2007; QUININO ET AL., 2010). Contudo, para que a contribuição desses profissionais seja viável, faz-se necessário que os mesmos portem um olhar ampliado, não focado apenas na doença (CESARINO ET AL., 2014).

Um fato importante a se considerar é que houve uma consonância entre os resultados de alguns indicadores utilizados no presente estudo. Na dimensão 'estrutura', a disponibilidade de veículo para as ações do PCE e a existência de recursos materiais educativos e humanos suficientes para as ações de controle de esquistossomose apresentaram os piores resultados, enquanto, na dimensão 'processo', as atividades relativas ao apoio laboratorial não foram desenvolvidas. Tal fato evidencia um senso congênere dos gestores ou a prioridade que cada um deles estabelece 
para a realização das atividades propostas para o controle da esquistossomose.

Em tempo, cabe aqui ressaltar que a descentralização política, administrativa e financeira para os municípios e estados aumentou a autonomia das instâncias subnacionais e permitiu espaços de participação, bem como o surgimento de experiências inovadoras em programas sociais. Embora se almeje com a descentralização uma melhor alocação de recursos, com reflexos positivos nas ações de saúde, muitos problemas têm surgido no nível local. As decisões tomadas, as quais deveriam traduzir a ordem certa das prioridades, maximizando os benefícios dos recursos aplicados e obtendo maior controle e planejamento das atividades, muitas vezes não têm sido desenvolvidas nas esferas municipais (MATOS, 2007).

Observa-se que, após a adoção de estratégias do Programa Sanar pelos municípios não prioritários, no que tange ao controle da esquistossomose, os avanços alcançados são incipientes. Apesar de ser um modelo desenvolvido que pode se adequar à realidade local, os municípios objeto do estudo realizam as ações de controle da esquistossomose dentro de suas possibilidades, limitações e ainda de maneira precária. Tais achados se contrapõem ao que se tem verificado em relação aos municípios prioritários, os quais têm demonstrado melhorias significativas dos indicadores de doenças negligenciadas (PERNAMBUCO, 2013). A diferença entre os municípios pode se dar pelo fato de que, nessa estratégia, o estado, além de apoiar e assessorar os entes municipais, também tem atuado como executor das ações com o desenvolvimento de atividades intencionistas.

De acordo com Santos e Melo (2008), a descentralização da vigilância epidemiológica para os municípios ocorre de forma gradual. Porém, na verdade, esse processo representa muito mais uma desconcentração de ações, uma vez que a maioria dos municípios não está estruturada para assumir efetivamente a descentralização dessas ações. Devido às diferenças políticas regionais, alguns municípios avançam mais do que outros na implantação da descentralização. Do ponto de vista financeiro, em muitos municípios, os recursos são escassos ou mal utilizados, produzindo como consequência ações incipientes e de baixo impacto.

Estudo realizado por Albuquerque, Mota e Felisberto (2015) sugere que a descentralização das ações da vigilância epidemiológica para o nível municipal trouxe como aspecto positivo a aproximação do nível executor das ações à população e aos seus problemas de saúde, o que resulta numa maior responsabilização por parte da gestão municipal. No entanto, apesar de diversos estudos apontarem que a estratégia de descentralização das ações e dos recursos financeiros para o âmbito municipal tem proporcionado experiências bem-sucedidas, é importante ressaltar que o contexto local, como, por exemplo, o perfil do gestor municipal, influencia de forma direta nesse processo (BARBOSA ET AL., 2010). Assim sendo, novos estudos são necessários para se avaliar a influência dos determinantes contextuais sobre o grau de implantação das ações de controle de esquistossomose.

\section{Conclusões}

Apesar das dificuldades operacionais referidas pelos profissionais dos municípios estudados, foi possível perceber uma perspectiva no avanço das ações de controle da esquistossomose, principalmente devido à maior participação da atenção básica nas ações que anteriormente ficavam restritas à vigilância epidemiológica, mesmo sendo esse envolvimento da atenção básica normatizado como um componente necessário para o sucesso dos resultados do programa.

Percebe-se uma fragilidade nos municípios estudados quanto à malacologia e à realização de ações voltadas à educação em saúde. Esses fatores contribuem para o 
alcance de melhores resultados, uma vez que o monitoramento malacológico detém um maior controle do vetor causador da doença, e as atividades de educação em saúde possibilitam uma maior aproximação da população com a equipe de saúde, o que é de extrema importância para a doença em questão. A esquistossomose é uma doença endêmica que está intimamente ligada às condições socioambientais e necessita de conscientização sanitária de quem vive nas

\section{Referências}

\author{
ALBUQUERQUE, A. C.; MOTA, E. L. A.; \\ FELISBERTO, E. Descentralização das ações de \\ vigilância epidemiológica em Pernambuco, Brasil. Cad. \\ Saúde Pública, Rio de Janeiro, v. 31, n. 4, p. 861-873, abr. \\ 2015 .
}

BARBOSA, M. C. L. et al. Efeitos da descentralização das ações de vigilância epidemiológica para as equipes de saúde da família. Epidemiol Serv Saúde, Brasília, v. 19, p. $347-54$, set. 2010 .

BRASIL. Ministério da Saúde. Portaria n ${ }^{\circ} 1.399$, de 15 de dezembro de 1999. Regulamenta a NOB SUS 01/96 no que se refere às competências da União, estados, municípios e Distrito Federal, na área de epidemiologia e controle de doenças, define a sistemática de financiamento e dá outras providências. Diário Oficial [da] União. Brasília, DF, 15 dez. 1999. Disponível em: <http://www.funasa.gov.br/site/wp-content/files_mf/ Pm_1399_1999.pdf>. Acesso em: 16 nov. 2016.

Ministério da Saúde. Portaria n ${ }^{\circ} 1172$, de 15

de junho de 2004. Regulamenta a NOB SUS 01/96 no que se refere às competências da União, Estados, áreas de transmissão da doença.

Para fortalecer as ações, é de fundamental importância uma melhor estrutura, maior preocupação com a capacitação e a qualificação profissional, realização de inquéritos coproscópicos para mapeamento de áreas com maior risco ambiental para transmissão da doença, atividades de educação em saúde para as comunidades e continuidade das ações integradas entre vigilância e atenção básica.
Municípios e Distrito Federal, na área de Vigilância em Saúde, define a sistemática de financiamento e dá outras providências. Diário Oficial [da] União. Brasília, DF, 15 jun. 2004. Disponível em: <http://www.saude. mt.gov.br/cosat/arquivo/1829/vigilancia-e-a-saudedo-trabalhador>. Acesso em: 16 nov. 2016.

Ministério da Saúde. Secretaria de Atenção à Saúde. Departamento de Atenção Básica. Política nacional de atenção básica. Brasília, DF: Ministério da Saúde, 2006. (Série Pactos pela Saúde 2006; v. 4). Disponível em: <http://www.conselho.saude.gov.br/ webpacto/text_atencao.pdf>. Acesso em: 16 nov. 2016.

Ministério da Saúde. Secretaria de Atenção à Saúde. Departamento de atenção à saúde. Vigilância em saúde: Dengue, Esquistossomose, Hanseníase, Malária, Tracoma e Tuberculose. 2. ed. Brasília, DF: Ministério da Saúde, 2008. (Cadernos de atenção básica $n^{\circ}$ 21). Disponível em: <http://dab.saude.gov.br/docs/ publicacoes/cadernos_ab/abcad21.pdf>. Acesso em: 16 nov. 2016.

Ministério da Saúde. Secretaria de Vigilância em 
Saúde. Departamento de Vigilância Epidemiológica. Vigilância da Esquistossomose Mansoni: diretrizes técnicas. 4. ed. Brasília, DF: Ministério da Saúde, 2014.

CESARINO, M. B. et al. A difícil interface controle de vetores - atenção básica: inserção dos agentes de controle de vetores da dengue junto às equipes de saúde das unidades básicas no município de São José do Rio Preto, SP. Saúde soc., São Paulo, v. 23, n. 3, p. 1018-1032, set. 2014.

CHIARAVALLOTI NETO, A. O programa de controle do dengue em São José do Rio Preto, São Paulo, Brasil: dificuldades para a atuação dos agentes e adesão da população. Cad Saúde Pública, Rio de Janeiro, v. 23, n. 7, p. 1656-64, jul. 2007.

DIAS, G. A. R.; LOPES, M. M. B. Educação e saúde no cotidiano de enfermeiras da atenção primária. Rev Enferm UFSM, Santa Maria, v. 3, n. 3, p. 449-460, 2013.

DONABEDIAN, A. Evaluating the quality of medical care. Milbank Mem Fund Q, Nova York, v. 44, n. 3, p. 166-206, 1966.

Advantages and limitations of explicit criteria for assessing the quality of health care. Health and Society, New York, v. 59, p. 100-105, 1981.

FARIAS, G. C. F. Avaliação do grau de implantação do Programa de Controle da Esquistossomose no município de Tracunhaém, Zona da Mata. 2010. 92 f. Monografia (Gestão de Sistema e Serviço de Saúde) - Centro de Pesquisas Aggeu Magalhães, Fundação Oswaldo Cruz, Recife, 2010.

FAVRE, T. C. Avaliação das ações de controle da esquistossomose implementadas entre 1977 e 1996 na área endêmica de Pernambuco, Brasil. Rev. Soc. Bras. Med. Trop., Uberaba, v. 34, n. 6, p. 569-76, dez. 2001.

KATZ, N.; PEIXOTO, S. V. Critical analysis of the estimated number of Schistosomiasis mansoni carriers in Brazil. Rev. Soc. Bras. Med. Trop., Uberaba v. 33, p. 303-308, jun. 2000.

MATOS, C. A. Os descaminhos da implementação do
SUS em Rondônia: contexto de federalismo predatório, de reformas gerenciais e de maus governos na floresta. 2007. 197 f. Tese (Doutorado em Saúde Pública) Escola Nacional de Saúde Pública, Fundação Oswaldo Cruz, Rio de Janeiro, 2007.

MENEZES, M. J. R. Avaliação do sistema de vigilância epidemiológica da esquistossomose no Estado da Bahia. 2005. 175 f. Dissertação (Mestrado Profissional em Saúde Pública) -Escola Nacional de Saúde Pública, Fundação Oswaldo Cruz, Rio de Janeiro, 2005.

OMOTTO, C. A.; SANTINI, S. M. L.; ESTEVES, J. L. M. Controle da dengue: uma análise da implementação do PNCD e a relação do processo de trabalho na $16^{\mathrm{a}}$ RSA. Congresso Consad de Gestão Pública, 2. Porto Alegre: Consad, 2009.

\section{ORGANIZAÇÃO PANAMERICANA DA SAÚDE} (OPAS); ORGANIZAÇÃO MUNDIAL DA SAÚDE (OMS). Resolução CD49.R19 de 2 de outubro de 2009. Eliminação de doenças negligenciadas e outras infecções relacionadas à pobreza. $49^{\circ}$ Reunião do Conselho Diretor; $61^{a}$ Sessão do Comitê Regional. Washington, DC, 2 out. 2009. Disponível em: <http://www.paho.org/bra/index.php?option=com docman\&task=doc_view\&gid=900\&Itemid $=423>$. Acesso em: 16 nov. 2016.

PEREIRA, A. P. C. M.; SERVO, M. L. S. A. A enfermeira e a educação em saúde: estudo de uma realidade local. Rev. Baiana Saúde Pública, Salvador, v. 30, n. 1, p. 7-18, jan./jun. 2006.

PERNAMBUCO. Secretaria Estadual de Saúde. Secretaria Executiva de Vigilância em Saúde.

Esquistossomose: Guia operacional para redução em Pernambuco. Recife: Secretaria Estadual de Saúde, 2012

Secretaria Estadual de Saúde. Secretaria Executiva de Vigilância em Saúde. Guia de apoio operacional ao Sistema de Informação do Programa de Controle da Esquistossomose para os municípios do Estado de Pernambuco. Recife: Secretaria Estadual de Saúde, 2012b. Disponível em: <http://portal. saude.pe.gov.br/sites/portal.saude.pe.gov.br/files/ 
esquistossomose_-_guia_sispce_.pdf>. Acesso em: 17 nov. 2016.

. Secretaria Estadual de Saúde. Secretaria Executiva de Vigilância em Saúde. Programa SANAR: cadernos de monitoramento. Recife: Secretaria Estadual de Saúde, 2013. (Série A: Normas e Manuais Técnicos; v. 1). Disponível em: $<$ http://portal.saude. pe.gov.br/sites/portal.saude.pe.gov.br/files/caderno_ de_monitoramento_esquistossomose.pdf > . Acesso em: 17 nov. 2016.

Secretaria Estadual de Saúde. Secretaria Executiva de Vigilância em Saúde. Boletim epidemiológico da esquistossomose e geohelmintíases, Pernambuco, v. 5, mar. 2015. Disponível em: <http:// portal.saude.pe.gov.br/sites/portal.saude.pe.gov.br/ files/boletim_vs_2015_final.pdf>. Acesso em: 1 fev. 2017.

QUININO, L. R. M.; BARBOSA, C. S.; SAMICO, I. O programa de controle da esquistossomose em dois municípios da zona da mata de Pernambuco: uma análise de implantação. Rev. Bras. Saúde Mater. Infant., Recife, v. 10, n. 1, p. 536-544, nov. 2010.
ROLLEMBERG, C. V. V. et al. R. Aspectos

epidemiológicos e distribuição geográfica da esquistossomose e geohelmintos, no estado de Sergipe, de acordo com os dados do Programa de Controle da Esquistossomose. Rev Soc Bras Med Trop, Uberaba, v. 44, n. 1, p. 91-96, fev. 2011.

SANTOS, S. S. B. S.; MELO, C. M. M. Avaliação da descentralização da vigilância epidemiológica para a Equipe de Saúde da Família. Ciênc. saúde coletiva, Rio de Janeiro, v. 13, n. 6, p. 1923-1932, dez. 2008.

\section{WORLD HEALTH ORGANIZATION (WHO). The} control of schistosomiasis: WHO Technical Report Series, Report of the WHO Expert Committee, Geneva, n. $728,1985$.

\footnotetext{
Recebido para publicação em abril de 2016

Versão final em setembro de 2016

Conflito de interesses: inexistente

Suporte financeiro: não houve
} 